\section{Ocular sarcoidosis in Thailand}

\begin{abstract}
Purpose To determine the prevalence of pulmonary sarcoidosis in patients with uveitis from the University Referral Centre in Thailand and to report on their clinical characteristics.

Methods We performed a retrospective review of results of radiological examinations of 209 consecutive new patients, diagnosed with uveitis. In patients with signs of pulmonary sarcoidosis, we reviewed clinical characteristics including age, gender, laterality and anatomical location of uveitis, and complications.

Results From 209 chest X-ray (CXR) examinations, one patient exhibited radiological signs typical of stage 1 sarcoidosis. Chest CT of three patients with posterior multifocal chorioretinitis (PMC) revealed abnormalities suggesting the diagnosis of pulmonary sarcoidosis. All PMC patients were females older than $\mathbf{5 0}$ years; they had no pulmonary complaints and their CXRs were without abnormalities. Conclusion Ocular sarcoidosis is prevalent in Thailand and our findings suggest that possibly more patients with sarcoidosis would be identified if diagnosis was thought of and ancillary tests were performed.
\end{abstract}

Eye (2010) 24, 1669-1674; doi:10.1038/eye.2010.107; published online 6 August 2010

Keywords: uveitis; sarcoidosis; Thailand

\section{Introduction}

Sarcoidosis is a multi-systemic granulomatous disease that is characterised by noncaseating granulomas. The exact cause of this disease is still unknown, although it has been suggested that genetic, environmental factors, or infectious agents may act as triggers. ${ }^{1,2}$ Organs affected most often are the lungs, thoracic lymph nodes, skin, and eyes. Sarcoidosis affects people of all racial and ethnic groups and can occur at all
K Pathanapitoon' ${ }^{1}$, JHM Goossens ${ }^{2,3}$,

TC van Tilborg ${ }^{2,3}$, P Kunavisarut ${ }^{1}$,

J Choovuthayakorn ${ }^{1}$ and A Rothova ${ }^{2}$

ages, however, there is a great variety of incidence and prevalence throughout the world. In the United States, the majority of patients are Black with a prevalence of 40 per 100000 , compared with 5 per 100000 among White Europeans. ${ }^{1,3}$ In contrast to Japan, sarcoidosis is less common in India, South-East Asia, New Zealand, and mainland China. ${ }^{3}$ So far, it is not known whether the rarity of sarcoidosis in South-East Asia is genuine or whether sarcoidosis in this geographic area remains underdiagnosed. Prevalence of sarcoidosis among patients with uveitis in the West is $\sim 8-10 \%,{ }^{4}$ in Japan $13 \%,{ }^{5}$ and in India and Taiwan $4 \%, 4,6$ but virtually no patients with ocular sarcoidosis have been identified in mainland China. ${ }^{6,7}$

In Thailand, systemic sarcoidosis has rarely been diagnosed and so far, no cases with ocular sarcoidosis have been reported. ${ }^{8,9}$ In this study, we explore the possibility of pulmonary sarcoidosis in 209 new consecutive patients with uveitis and describe ocular features of four Thai patients with signs of associated pulmonary sarcoidosis.

\section{Materials and methods}

We performed a retrospective review of the radiological examinations of 209 consecutive new human immunodeficiency virus-negative patients diagnosed with uveitis in Chiang Mai University Hospital between June 2006 and December 2008.

All patients with uveitis underwent a screening protocol, which included chest X-ray (CXR) and various laboratory tests, which encompassed erythrocyte sedimentation rate and complete blood counts, serology for human immunodeficiency virus, Treponema pallidum, Toxoplasma gondii, and human leukocyte antigen (HLA-B27) typing. In addition to the initial screening, a chest CT was performed in all patients with posterior multifocal choroiditis ${ }^{10}$ (PMC, 3/209, 1.4\%) and all with chronic vitritis in patients older than 50 years $(4 / 209 ; 1.9 \%$; Figure 1). Uveitis was classified according to the
${ }^{1}$ Department of Ophthalmology, Faculty of Medicine, Chiang Mai University, Chiang Mai, Thailand

${ }^{2}$ Uveitis Center, Department of Ophthalmology, University Medical Center Utrecht, Utrecht, The Netherlands

Correspondence: K Pathanapitoon, Department of Ophthalmology, Faculty of Medicine, 110 Intawaroros Road, Chiang Mai 50200, Thailand Tel: + 6653 945512-3; Fax: + 6653946121 .

E-mail: kpathana@ mail.med.cmu.ac.th ${ }^{3}$ These authors contributed equally to this work

Received: 9 February 2010 Accepted in revised form: 19 June 2010 Published online: 6 August 2010 


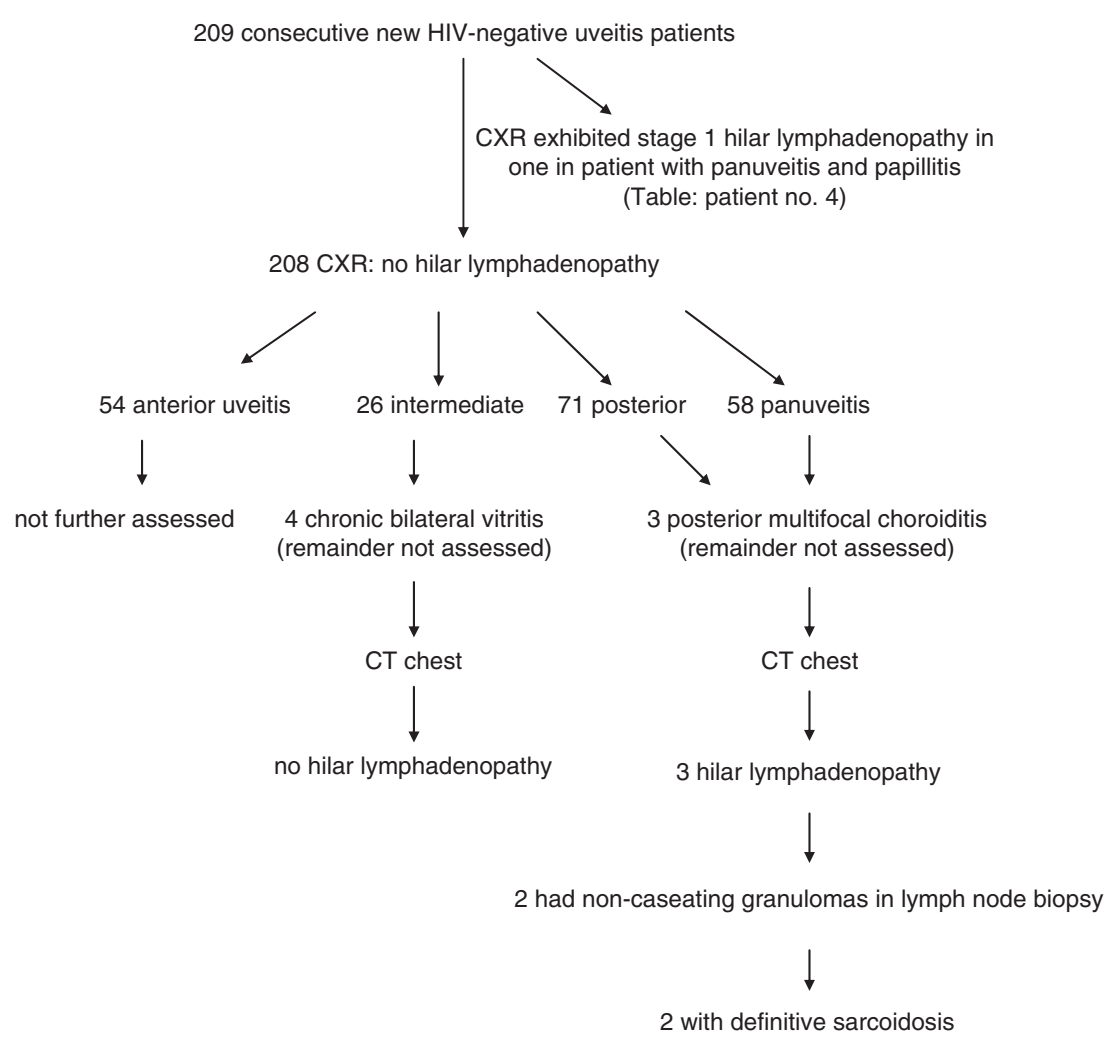

Figure 1 Flow chart of patients with uveitis documenting the chest examinations performed.

anatomical localisation as recommended by the SUN working group. ${ }^{11}$

The purified protein derivative standard test was performed in 23 patients and included all with abnormal CXRs suggesting (old) tuberculosis (TB; $n=6)$ or sarcoidosis $(n=1)$, abnormal chest CT suggesting sarcoidosis $(n=4)$, clinical presentation of choroditis $(n=5)$, or retinal vasculitis $(n=7)$. Tissue biopsy was performed in two patients with PMC and normal CXR, but a chest CT suggesting sarcoidosis. The remaining two patients with signs of pulmonary sarcoidosis (one on CXR and one on CT scan) refused the tissue sampling.

We also reviewed the ocular characteristics of patients with signs of pulmonary sarcoidosis revealed by radiological and chest $\mathrm{CT}$, which included age, gender, laterality, anatomical location of uveitis, ocular manifestation, visual acuity, and complications.

\section{Results}

A total of 209 patients with uveitis were included in this study; 102 (49\%) men and 107 (51\%) women with average age of 40 years (range $8-85$ years). Out of the total of 209 patients, 54 had anterior uveitis, 26 had intermediate uveitis, 71 had posterior, and 58 had panuveitis (Figure 1). In all, 1 out of 209 patients (0.5\%) had bilateral hilar lymphadenopathy typical of sarcoidosis stage 1 . This 38-year-old male patient presented with blurred vision due to acute unilateral papillitis and with inflammatory reaction in the anterior segment and vitreous (Table 1). He had no systemic symptoms.

A chest CT was performed in seven patients who presented either with PMC ( $n=3$, all females) or chronic idiopathic vitritis in patients older than 50 years $(n=4$, all females). Of these seven chest CTs, three patients with PMC exhibited bilateral abnormalities typical of the diagnosis of sarcoidosis (Table 1). All had bilateral hilar adenopathy, sometimes in combination with enlarged lymph nodes in paratracheal or other thoracal locations. Four elderly females with bilateral vitritis, but without PMC, had no abnormalities on chest CT and their definitive diagnoses were not clarified.

In two patients with PMC and positive CT scan, tissue biopsy from paratracheal lymph nodes was performed and revealed in both, noncaseating granulomas, negative for acid-fast bacilli with peroidic acid-Schiff and Gomori methenamine stains. All four patients with signs of pulmonary sarcoidosis had negative tuberculin skin tests. All four patients with suspected sarcoidosis were seen by a pulmonologist and TB was ruled out. According to the new diagnostic criteria for ocular 


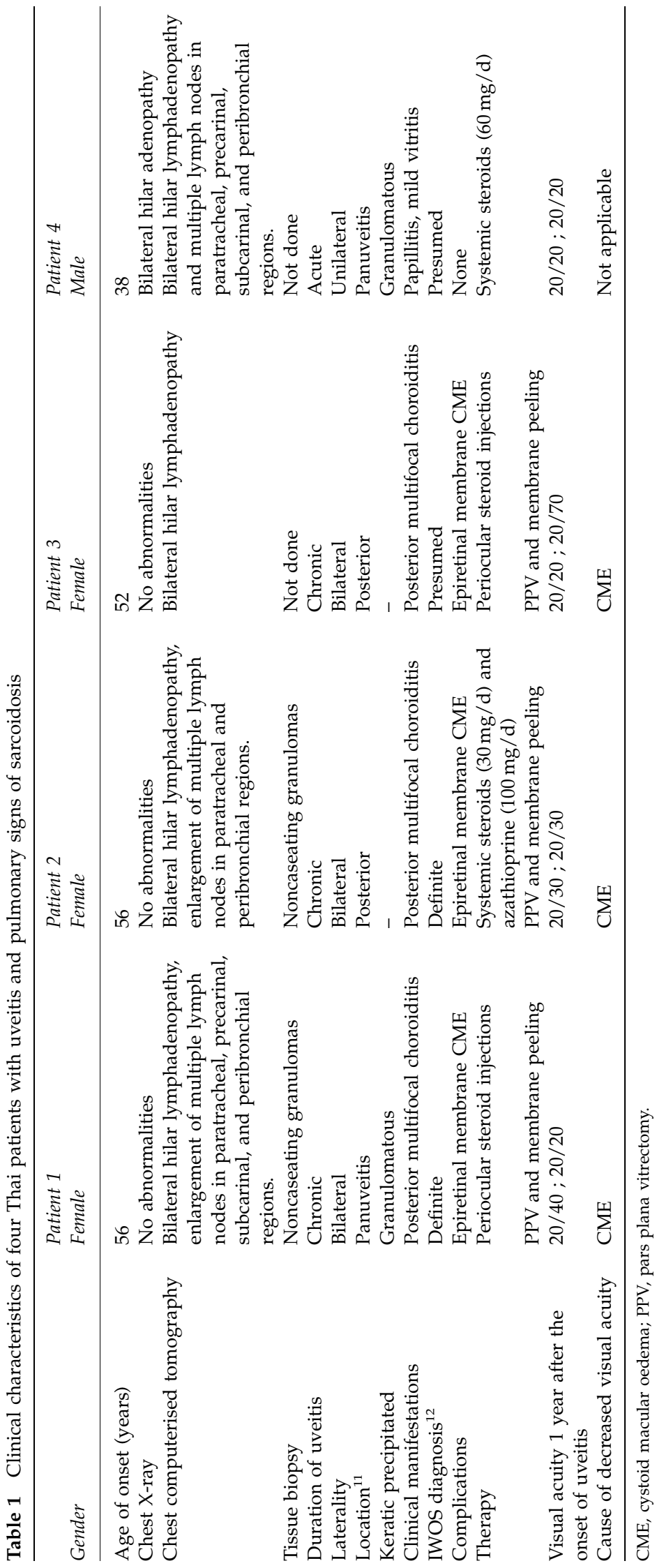




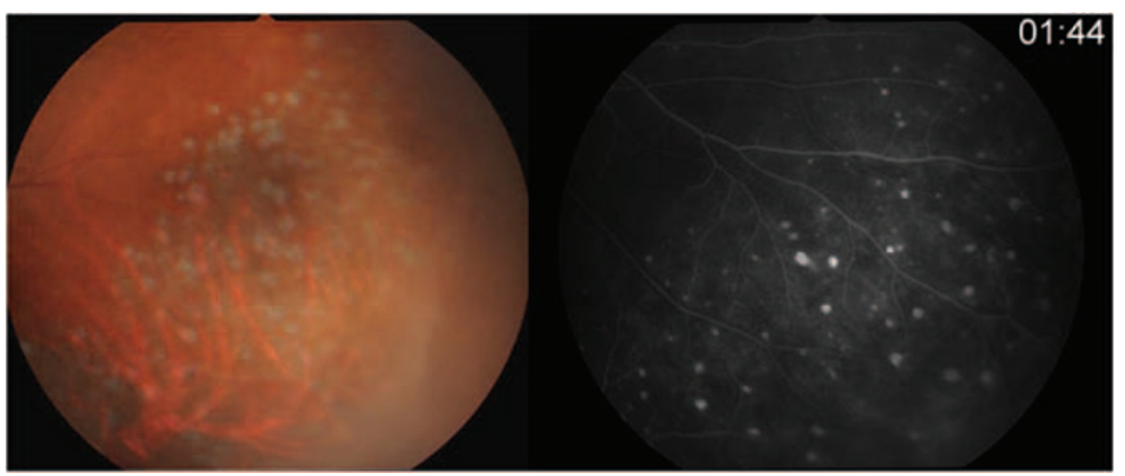

Figure 2 Fundus and fluorescein angiography photographs demonstrate multiple chorioretinal punched out lesions in the peripheral retina in elderly female Thai patients with hilar adenopathy on chest CT and histological diagnosis of sarcoidosis (Table 1, patient no 2).

sarcoidosis, two of our four patients fulfilled the criteria for a definitive and other two for a presumed diagnosis of ocular sarcoidosis. ${ }^{12}$

All three patients with positive chest CT findings were females aged older than 50 years who suffered from chronic PMC characterised by bilateral vitritis associated with peripheral retinal punched-out lesions (Table 1 and Figure 2). All developed cystoid macular oedema (CME) and epiretinal membranes (Table 1). After pars plana vitrectomy and membrane peeling their CME improved and their visual acuities fluctuated between 20/70 and 20/20 (Table 1).

In addition, 1 out of 209 patients $(0.5 \%)$ was diagnosed with active pulmonary TB and TB-associated uveitis (choroidal granuloma with exudative retinal detachment).

\section{Discussion}

Our study documents that pulmonary sarcoidosis is prevalent in the uveitis population in Thailand as 1 out of $209(0.5 \%)$ new consecutive patients with uveitis had radiological signs of sarcoidosis, and 3 additional patients exhibited bilateral hilar adenopathy typical of sarcoidosis on their chest CT. In two patients with an abnormal chest CT, tissue biopsy confirmed the diagnosis of definitive sarcoidosis. The patient with an abnormal CXR was a 38-year-old male with unilateral panuveitis and papillitis, and three patients with negative CXR, but positive chest $\mathrm{CT}$, were elderly females with chronic bilateral PMC.

In the West, the prevalence of sarcoidosis among the patients with uveitis is $\sim 7 \% .^{3}$ Our results indicate that at least 4 out of 209 (2\%) uveitis patients suffered from pulmonary sarcoidosis. This percentage might be in reality even higher, if additional diagnostic tests such as chest CT were systematically applied. In Japan and China, a large increase in ocular sarcoidosis was noted over time. ${ }^{5,6}$ It is not clear whether this increase is because of previous underdiagnosis or concerns a genuine increase of prevalence of ocular involvement. Sarcoidosis can remain subclinical for long periods of time and may also be mistaken for TB. It is possible, that sarcoidosis in Thailand might be unrecognised and/ or confused with TB; a similar phenomenon was also reported from India. ${ }^{13}$

The current major diagnostic test for the diagnosis of sarcoidosis is undoubtedly CXR, but for the definitive diagnosis of sarcoidosis a confirmation by tissue biopsy is required. In our study, tissue biopsy was attempted in two patients with PMC and confirmed the diagnosis in both. Unfortunately, because of nonmedical reasons, tissue diagnosis could not be obtained in the remaining two patients with pulmonary abnormalities and their diagnoses remained presumed. ${ }^{12}$ The value of CXR for diagnosis of sarcoidosis in patients with uveitis was repeatedly debated, and it is currently agreed that the chest $\mathrm{CT}$ is more sensitive than the CXR. ${ }^{2,14,15}$ In addition, serum ACE and lysozyme levels are useful for the screening of uveitis patients, ${ }^{1,16}$ however, these serological tests were not available in our institute. It is agreed that chest CT is superior and provides more details for assessing the pattern and extent of the disease compared with CXR. ${ }^{2,14,15}$ Possibly, the systematic making of a chest $\mathrm{CT}$ in patients with a clinical suspicion of sarcoidosis might reveal additional cases. However, chest CT is not recommended for the initial screening of patients with uveitis for sarcoidosis, because of its high radiation and cost. ${ }^{1}$ So far, studies systematically comparing diagnostic values of CT and CXR in uveitis are lacking and the indications for chest $\mathrm{CT}$ in patients with uveitis are not clear. It was reported that especially elderly females with PMC had frequently positive chest CT findings typical of sarcoidosis. ${ }^{10,15}$ This observation is entirely in agreement with our findings.

The overall value of CXR in the initial screening for uveitis in Northern Thailand might be considered limited as only 2 out of 209 CXR (1\%) were informative for the 
diagnosis of ocular diseases (one sarcoidosis and one active TB). The limited value of CXR in initial screening for uveitis was also reported in other series. ${ }^{2,14,15}$ Future studies are needed to indicate the value of CXR in the initial screening of all patients with uveitis and to determine the exact role of chest $\mathrm{CT}$ in diagnostic workup of patients with uveitis and presumed sarcoidosis.

The typical features of ocular sarcoidosis include uveitis (30-70\%) and conjunctival nodules (40\%). ${ }^{17}$ Posterior uveitis with the involvement of the retina was most frequently seen in elderly White females, whereas anterior uveitis was noted mainly in studies where the majority of the patients were Black. ${ }^{17}$ All of our patients had posterior segment involvement (100\%), however, this might reflect a selection bias as chest CT was performed only in seven patients with bilateral PMC and/or vitritis. In Japan, posterior segment involvement was documented in more than half of the patients with ocular sarcoidosis. ${ }^{18} \mathrm{PMC}$ is a clinical entity primarily encountered in White elderly females. It is characterised by the presence of multiple, small, round, punched-out lesions in the peripheral retina and an associated intraocular inflammatory reaction. ${ }^{10}$ Approximately onethird of PMC patients had systemic sarcoidosis and the majority developed CME. ${ }^{10,17,19}$ Similar manifestations were present in our three patients. Visual loss in sarcoidosis was predominantly noted in patients with chronic posterior uveitis and was mainly caused by CME $(19-72 \%) .{ }^{17}$ In India, however, CME was much less frequently found $(7 \%) .^{13}$

Differential diagnosis of ocular sarcoidosis includes mainly lymphoma and TB. The diagnosis of lymphoma was considered in all of our patients, however, none had further symptoms or manifestations of lymphoma; in addition, all patients were assessed by a pulmonologist. Two patients had definitive sarcoidosis diagnosed by tissue biopsy. No signs of lymphoma were noted in two patients with presumed sarcoidosis. The male patient (no 4) with presumed sarcoidosis had acute uveitis, which completely healed following a systemic course with corticosteroids and the hilar lymphadenopathy showed no further progression at 1-year follow-up. The remaining patient with presumed sarcoidosis and PMC reacted well to periocular corticosteroid injections. No progression of ocular or systemic features was observed at 1-year follow-up. Ocular TB in the wake of active pulmonary infection was diagnosed in 1 out of $209(0.5 \%)$ human immunodeficiency virus-negative uveitis patients. In our previous study, we identified presumed ocular TB in 3out of 138 (2\%) of new uveitis patients who were human immunodeficiency virus-negative. ${ }^{8}$ Recently, the association between latent TB and uveitis was described. ${ }^{20}$ The interferon gamma release assay such as the Quantiferon test was not used in our series, and therefore, the number of patients with uveitis, which might have been related to latent $\mathrm{TB}$, was not identified.

Our study has all the shortcomings of a retrospective study. It was not possible to perform chest CT scans in all patients with clinical features compatible with the diagnosis of sarcoidosis (eg, all forms of chronic uveitis including vasculitis). We performed chest CTs only in seven patients (elderly with chronic vitritis and all with PMC) and in consequence, the prevalence of sarcoidosis observed by us is probably underestimated. Because of nonmedical causes, we were not able to perform the review of systems and other ancillary tests in all patients with uveitis.

In conclusion, our study confirms that sarcoidosis is prevalent in Thailand and points out that possibly more patients with sarcoidosis would be identified among the uveitis patients in the Far East, if the diagnosis was considered and ancillary tests performed. Future investigations are needed to clarify the exact prevalence, clinical spectrum and visual prognosis of ocular sarcoidosis in South-East Asia.

\section{Summary}

What was known before

- Sarcoidosis is prevalent among patients with uveitis in the West.

What this study adds

- Ocular sarcoidosis is prevalent in Thailand. And possibly more patients with sarcoidosis would be identified among the uveitis patients in Far East, if the diagnosis was considered and ancillary tests performed.

\section{Conflict of interest}

The authors declare no conflict of interest.

\section{Acknowledgements}

Juntima Euathrongchit MD for her advice in evaluation of radiological examinations.

Funding: Supported in part by the combined grants from Dr P Binkhorst Foundation for Ophthalmologic research, Nijmegen; Landelijke stichting voor Blinden en Slechtzienden, Utrecht; Rotterdamse Vereniging Blindenbelangen, Rotterdam; Stichting voor Ooglijders, Rotterdam; Katholieke Stichting voor Blinden en Slechtzienden, Grave, Stichting Oog, 's Gravenzande, and Dr FP Fischer Stichting, Amersfoort, the Netherlands.

\section{References}

1 Michael C. Sarcoidosis. N Engl J Med 2007; 357: 2153-2165.

2 Margolis R, Lowder CY. Sarcoidosis. Curr Opin Ophthalmol 2007; 18: 470-475. 
3 Sharma OP. Sarcoidosis around the world. Clin Chest Med 2008; 29: 357-363.

4 Rathinam SR. Global variation and pattern changes in epidemiology of uveitis. Indian J Ophthalmol 2007; 55: 173-183.

5 Goto H, Mochizuki M, Yamaki K, Kotake S, Usui M, Ohno S. Epidemiological survey of intraocular inflammation in Japan. Jpn J Ophthalmol 2007; 51: 41-44.

6 Chung YM. Uveitis with biopsy-proven sarcoidosis in Chinese-a study of 60 patients in uveitis clinic over a period of 20 years. J Chin Med Assoc 2007; 70: 492-496.

7 Yang P, Zhang Z, Zhou H, Li B, Huang X, Gao Y et al. Clinical patterns and characteristics of uveitis in a tertiary center for uveitis in China. Curr Eye Res 2005; 30: 943-948.

8 Pathanapitoon K, Kunavisarut P, Ausayakhun S, Sirirungsi W, Rothova A. Uveitis in a tertiary ophthalmology centre in Thailand. Br J Ophthalmol 2008; 92: 474-478.

9 Manonukul J, Wanitphakdeedecha R, Wisuthsarewong W, Thirapote P. Histopathologic aid to diagnosis of sarcoidosis: report of 8 cases. J Med Assoc Thai 2006; 89: 864-871.

10 Lardenoye CW, Van der Lelij A, de Loos WS, Treffers WF, Rothova A. Peripheral multifocal chorioretinitis: a distinct clinical entity? Ophthalmology 1997; 104: 1820-1826.

11 Standardization of uveitis nomenclature (SUN) working group. Standardization of uveitis nomenclature for reporting clinical data. Results of the first international workshop. Am J Ophthalmol 2005; 140: 509-516.
12 Herbort CP, Rao NA, Mochizuki M, members of Scientific Committee of First International Workshop on Ocular Sarcoidosis. International criteria for the diagnosis of ocular sarcoidosis: results of the first International Workshop On Ocular Sarcoidosis (IWOS). Ocul Immunol Inflamm 2009; 17: 160-169.

13 Khanna A, Sidhu U, Bajwa G, Malhotra V. Pattern of ocular manifestations in patients with sarcoidosis in developing countries. Acta Ophthalmol Scans 2007; 85: 609-612.

14 Lynch III JP. Computed tomographic scanning in sarcoidosis. Semin Respir Crit Care Med 2003; 24: 393-418.

15 Kaiser PK, Lowder CY, Sullivan P, Sanislo SR, Kosmorsky GS, Meziane MA et al. Chest computerized tomography in the evaluation of uveitis in elderly women. Am J Ophthalmol 2002; 133: 499-505.

16 Kawaguchi T, Hanada A, Horie S, Sugamoto Y, Sugita S, Mochizuki M. Evaluation of characteristic ocular signs and systemic investigations in ocular sarcoidosis patients. Jpn J Ophthalmol 2007; 51: 121-126.

17 Rothova A. Ocular involvement in sarcoidosis. $\mathrm{Br} \mathrm{J}$ Ophthalmol 2000; 84: 110-116.

18 Ohara K. Intraocular manifestations of systemic sarcoidosis. Jpn J Ophthalmol 1992; 36: 452-457.

19 Abad S, Meyssonier V, Allali J, Gouya H, Giraudet AL, Monnet D et al. Association of peripheral multifocal choroiditis with sarcoidosis: a study of thirty-seven patients. Arthritis Rheum 2004; 51: 974-982.

20 Tabbara KF. Tuberculosis. Curr Opin Ophthalmol 2007; 18: 493-501. 